\title{
Numerical Simulation of Mass Ratio's Effect on Vortex-Induced Vibration of Suspended Submarine Pipeline
}

\author{
E. J. Zhao, B. Shi, J. Zhang \& K. Cao \\ College of Engineering \\ Ocean University of China \\ Qingdao, China
}

\begin{abstract}
Submarine Pipeline is used widely in ocean engineering now. In order to study the mass ratio's effect on the submarine pipelines' vortex-induced vibration, two-degrees-of-freedom vortex-induced vibrations of the pipelines with large and small mass ratio are simulated with the software ANSYS-CFX. When the mass ratios are 7.08 and 3.2, the lock-in, beat and phase switch phenomena were caught in the experiment. The effects of mass ratio on VIV were discussed. Besides, the trajectories were observed with different mass ratios. The research provides a theoretical basis for engineering practice.
\end{abstract}

Keywords-VIV; CFX; frequency; phase switch; beat; trajectory

\section{INTRODUCTION}

The development of the deep water oil resources becomes a consensus among all countries in the world, the usage of the pipeline is becoming more and more widespread. Therefore, the vortex-induced vibration(VIV) research of the submarine pipeline is being paid more attention to.

Representative experimental studies on VIV include those of Griffin and Koopmann[1], Brika and Laneville[2] and Hover et al.[3], in which classic lock-in was observed, whereas they did not consider the situation of the shedding frequency coincided with the natural structure frequency. However, recently, the experimental results of Gharib[4] and Khalak [5] provided examples of significant flow-induced vibration without lock-in and suggested that the lock-in of VIV is dependent on the values of the cylinder/fluid mass ratio. So the mass ratio is a very important factor to VIV.

On the basis of the previous studies, the vortex-induced vibration(VIV) of suspended submarine pipeline is simulated using ANSYS-CFX software.

\section{NUMERICAL MODELING}

Hydrodynamic load problem of submarine pipeline is very complex. When the pipeline diameter is much smaller than the incident wave length, it can be considered the structure only cause local perturbations in the flow field near pipeline and the pipeline induces little effect on the wave diffraction. The Morison formula calculates the horizontal force $F_{k}$ and lift force $F_{l}$ of pipeline.

$$
\begin{gathered}
F_{h}=F_{d}+F_{m}=\frac{1}{2} C_{d} \rho D u|u|+C_{m} \rho \frac{\pi D^{2}}{4} \frac{\partial u}{\partial t} \\
F_{l}=\frac{1}{2} C_{l} \rho D u^{2}
\end{gathered}
$$

$C_{d}$ is the drag coefficient; $C_{m}$ is quality factor; $C_{l}$ is lift coefficient; $\rho$ is fluid density; $D$ is pipe diameter; $u$ is flow velocity; $\frac{\partial u}{\partial t}$ is the flow acceleration.

Based on the above theory, the 3D flow field mesh model was established by software ANSYS-CFD(see, Fig.1.). The calculation area is $30 \mathrm{D} \times 20 \mathrm{D} \times 5 \mathrm{D}$ ( $\mathrm{D}$ is the diameter of submarine pipeline). In the current entrance boundary, the given speed value is $u=0.02 \mathrm{~m} / \mathrm{s}, \quad v=w=0 \mathrm{~m} / \mathrm{s}$, pressure value is $p=0$; In outlet boundary, the derivative of the flow parameters is zero, even pressure and pressure gradient values are zero; the upper and lower side of the fluid calculation region adopt free slip wall and the wall shear stress is 0 . That is meaning flow velocity near the wall is not affected by wall friction. The left and right boundary of the region are symmetry walls; The cylindrical wall is interface wall. the entrance condition of boundary is the initial condition of calculation.

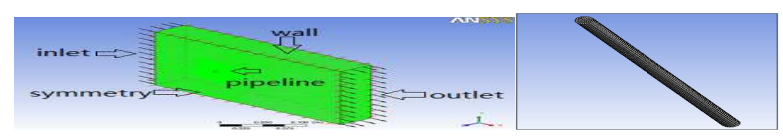

FIGURE I. FLUID MESH ANALYSIS MODEL DIAGRAM AND THE CYLINDER MODEL

\section{Flow ANALYSIS AROUND FIXED CYLINDER}

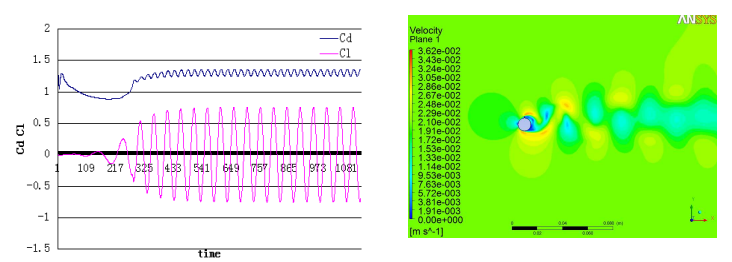

FIGURE II. CL AND CD UNDER RE $=200$ AND THE FLOW FIELD AROUND PIPELINE 
Fig.2. shows the flow lift coefficient and drag coefficient curve when the Re is 200 and the flow field around pipeline. As can be seen from the graph, the drag coefficient $\mathrm{Cd}$ increased gradually with the time step increasing and stabilized at about 1.30, the lift coefficient amplitude stabilized at about 0.7. Comparing with the previous classical results, the result has enough accuracy in table 1, this ensures the simulation accuracy of fluid-structure interaction (FSI) calculation in the following work.

TABLE I. RESUlts OF Flow AROUND CirCULAR CyLindER(RE=200)

\begin{tabular}{|c|l|l|l|}
\hline $\boldsymbol{R e}=\mathbf{2 0 0}$ & $\boldsymbol{C}_{\boldsymbol{d}}$ & $\boldsymbol{C}_{\boldsymbol{l}}$ & \multicolumn{1}{c|}{$\boldsymbol{S} \boldsymbol{t}$} \\
\hline Braza M(1985Num) & 1.31 & 0.65 & 0.19 \\
\hline Lecointe(1989Num) & 1.29 & 0.60 & 0.19 \\
\hline Marinez(1979Exp) & 1.30 & 0.70 & 0.19 \\
\hline Wei Zhili(2006 Num) & 1.29 & 0.74 & 0.18 \\
\hline result & 1.30 & 0.70 & 0.193 \\
\hline
\end{tabular}

\section{VORTEX-INDUCED VIBRATION ANALYSIS OF DIFFERENT} MASS RATIOS

\section{A. Two Important Phenomena with Different Mass Ratios}

"Beat" is a very uncommon physical phenomena of vortex-induced vibration. When the pipeline natural frequency and the vortex-induced vibration frequency are close, the displacement response and lift coefficient values are decreasing with the time increasing.. When the reduced velocity is 3 and mass ratio is 7, the "Beat" phenomenon is captured. As can be seen in Fig.3., this is one of the most important phenomena in vortex-induced vibration.

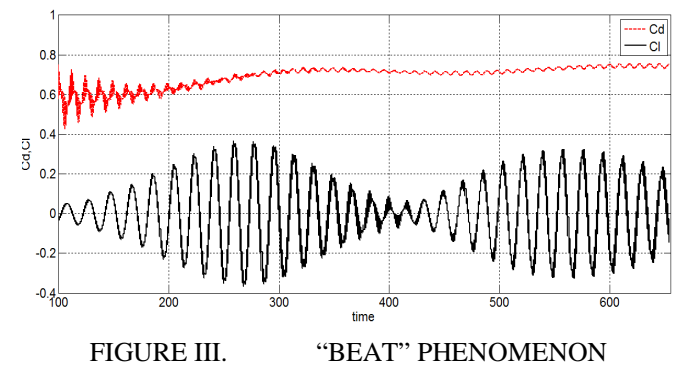

Another important phenomenon is called "phase switch", referring to the lift coefficient and displacement response curve phase change from "in phase"to "reverse phase". When the mass ratio is high, the phenomenon was not found in the locking area and non-locking region. However, when the mass ratio was 3.2 and the reduced velocity reached 7.05, the "phase switch" phenomenon was observed. In Fig.4., solid line represents the lateral displacement curve and the dotted line represents the lift coefficient curve. The lateral displacement response phase of structure was contrary to the lift coefficient phase. Because of the damping of the system, the flow load change can not consist with structure displacement change which caused the " phase switch" phenomenon.

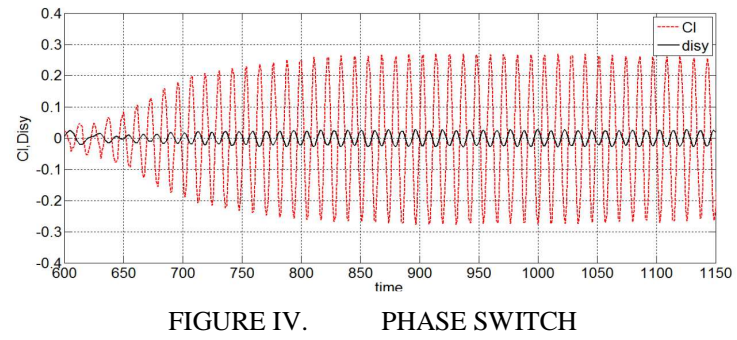

\section{B. Power Spectra with Different Mass Ratios}

When the mass ratio is 3.2 , the first-order natural frequency of pipeline is $6.917 \mathrm{~Hz}$ and the reduced velocity range is about 5-9 in the locking area.(see Fig. 5.)

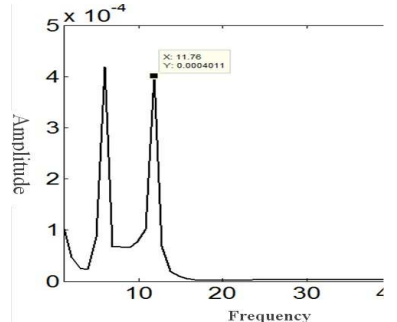

(a)Fair current



(b)Transverse
FIGURE V. POWER SPECTRA OF DISPLACEMENT UNDER UR=5 AND MASS RATIO IS 3.24

When the mass ratio is 7.08 , the reduced velocity range is about 3-6 in the locking area. In the case of mass ratio is 3, when reduced velocity is less than 4 , the vibration frequency is two times the frequency of lateral vibration. When the reduced velocity is 5 , the vibration characteristics present horizontal vibration frequency changing, first to the two times the transverse vibration frequency, and then the same to the transverse vibration frequency. The characteristic is the same under greater reduced velocity. But when the mass ratio is 7.08 , horizontal vibration frequency is always two times transverse vibration frequency. This shows the mass ratio not only influences the range of lock-in, but also effects the horizontal vibration frequency.(see, Fig.6.)

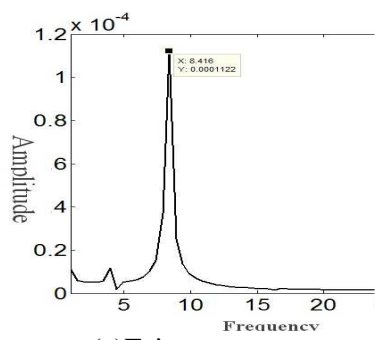

(a)Fair current

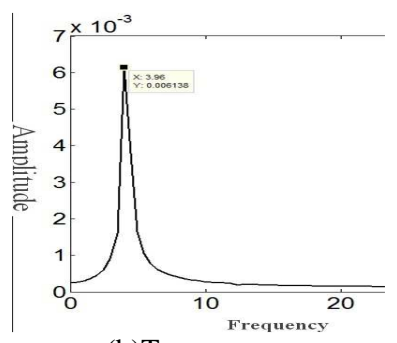

(b)Transverse

FIGURE VI. POWER SPECTRA OF DISPLACEMENT UNDER $\mathrm{UR}=5$ AND MASS RATIO IS 7

\section{Track Analysis with Different Mass Ratios}

The new theory phenomenon is that the frequency of the in-line VIV of the cylinder is equal to that of the cross-flow VIV and the VIV trace is the oval. 

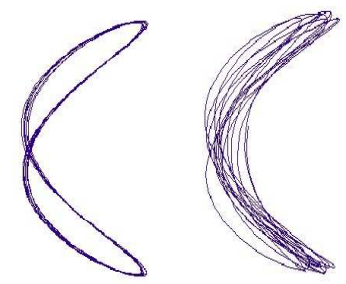
(a) $u_{r}=4$
(b) $u_{r}=5$
FIGURE VII. ORIES UNDER SMALL RATIO
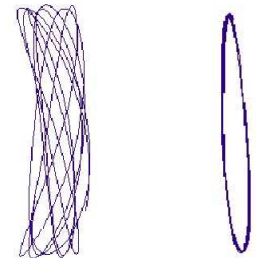

(a) $u_{r}=4$

(b) $u_{r}=5$

FIGURE VIII. TRAJECT ORIES UNDER HIGH RATIO

When the mass ratio is 3 and the reduced velocity $u_{r=4}$ and 5 , the trajectory is a shape " 8 ", the two direction vibration influence each other, and when the transverse vibration amplitude value reached the maximum value, the horizontal vibration amplitude value basically reached the maximum value. Besides, the transverse vibration and horizontal vibration achieved the balance position in the same time, and there is no phase difference between both.(see, Fig.7). When

the mass ratio is 9 and $u_{r=4}$ and 5 , the trajectory shape is oval which is different with shape " 8 ". When the transverse vibration amplitude value reached the maximum value, the horizontal vibration basically reached the balance position. There is the phase difference of 90 degrees between both(see, Fig.8).

\section{V.CONCLUSION}

In this paper, through CFX fluid-solid coupling scheme, the vortex-induced vibration characteristics of pipeline were studied by simulation. The change of power spectrum, amplitude, lift coefficient and trajectory were discovered with different mass ratio. Two important phenomena of vortex-induced vibration were found which were "Beat" and "phase switch". The trajectories were also different between large and small mass ratios. This provides the certain theory basis for the further research of the vortex-induced vibration about pipeline.

ACKNOWLEDGMENT: This study was financially supported by National Nature Science Fund of China (Grant No. 51279189; 50879084).

\section{REFERENCE}

[1] O.M. Griffin, G.H. Koopmann. The vortex-exited lift and reaction forces on resonantly vibrating cylinders. Journal of Sound and Vibration,54, pp. 435-438, 1977.

[2] D. Brika, A. Laneville. Vortex-induced vibration of a long flexible circular cylinder. Journal of Fluid Mechanics, 250, pp.481-508,1993.

[3] F.S. Hover, S.N. Miller, M.S. Triantafyllou. Vortex-induced vibration of marine cables: experiments using force feedback. Journal of Fluid and Structures,11, pp. 307-326,1997.

[4] M.R. Gharib. Vortex-induced vibration, absence of lock-in and fluid force deduction. California Institute of Technology, Pasadena, CA, USA, 1999.

[5] A. Khalak, C.H.K. Williamson. Fluid forces and dynamics of a hydroelastic structure with very low mass and damping. Journal of Fluid and Structures,11, pp.973-982,1997. 